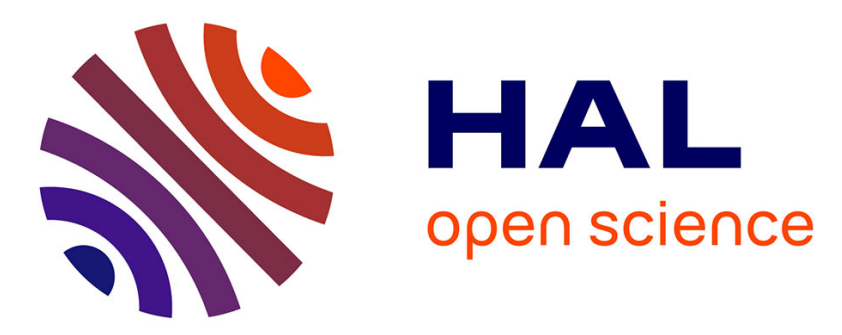

\title{
On dialogue modeling: a dynamic epistemic inquisitive approach
}

Maria Boritchev, Philippe de Groote

\section{To cite this version:}

Maria Boritchev, Philippe de Groote. On dialogue modeling: a dynamic epistemic inquisitive approach. New Frontiers in Artificial Intelligence. JSAI-isAI 2020., Nov 2020, Online, Japan. pp.21-36, 10.1007/978-3-030-79942-7_2 . hal-03065236

\section{HAL Id: hal-03065236 \\ https://hal.inria.fr/hal-03065236}

Submitted on 14 Dec 2020

HAL is a multi-disciplinary open access archive for the deposit and dissemination of scientific research documents, whether they are published or not. The documents may come from teaching and research institutions in France or abroad, or from public or private research centers.
L'archive ouverte pluridisciplinaire HAL, est destinée au dépôt et à la diffusion de documents scientifiques de niveau recherche, publiés ou non, émanant des établissements d'enseignement et de recherche français ou étrangers, des laboratoires publics ou privés. 


\title{
On dialogue modeling: a dynamic epistemic inquisitive approach
}

\author{
Maria Boritchev and Philippe de Groote \\ LORIA, UMR 7503, Université de Lorraine, CNRS, Inria, 54000 Nancy, France
}

\begin{abstract}
This paper introduces a formal model of dialogue based on insights and ideas developed by Jonathan Ginzburg in [11]. This model, which is logic based, takes advantage of inquisitive semantics [4], which allows to model both declarative and interrogative sentences in a uniform way. It appeals to ideas derived from classical epistemic logic in order to model the knowledge states of the dialogue participants, and includes a context-updating mechanisms based on the type-theoretic dynamic logic developed in [15].
\end{abstract}

\section{Introduction}

Dialogues are build in a dynamic way. An utterance follows another and may contain references to concepts and language constructions introduced by previous utterances, but also by the context of the conversation. This dialogue context is constantly updated as the dialogue unrolls, both for each dialogue participant, privately, and in a public way, building the common ground [18], composed of information that is available to everyone equally (participants and possible audience). Consider the following piece of dialogue (part of the example we present Section 6):

(1) [CONTEXT: Albert and Bernard would like to know when is Cheryl's birthday. She gives them some clues that might help them guess the date.]

a. Cheryl (to Albert): Can you figure it out now?

b. Albert (to Cheryl): I don't know when your birthday is, but I know Bernard doesn't know, either.

This simple excerpt illustrates several features that are characteristic of dialogue. It stresses the importance of the context, and in particular, of keeping track of the issues that are being raised, of what questions are under discussion. This allows, for instance, the pronominal anaphoric "it" in (1-a) to be resolved. It also demonstrates that a dialogue is not only made of declarative sentences, but also of interrogative ones (direct, as in (1-a), or embedded under a propositional attitude verb, as in (1-b)). Consequently, the development of a logic-based formal model of dialogue requires a logic that can express the semantic content of both declaratives and interrogatives. Inquisitive logic [4] is such a logic.

Example (1) also demonstrates the need for a dialogue model to integrate epistemic modalities. In (1-b), Albert mentions his (private) knowledge about Bernard's (private) knowledge. Therefore, we need to model the knowledge states of the dialogue 
participants; see Section 4. The whole dialogue is then modeled using a dialogue gameboard, see Section 2. We showcase the way our model works on an example in English, Cheryl's birthday problem, a logical puzzle that went viral on the internet of few years ago $^{1}$, see Section 6 . We then discuss and compare our work to related approaches such as [8] and [5].

Consider Figure 1, which represents a dynamic view of a dialogue divided in negotiation phases. A negotiation phase corresponds to the discussion by the participants of one issue; it begins with the introduction of this issue and ends when an agreement has been reached (while this agreement can be to drop the issue, to disagree). The result of the negotiation phase is then stored in the dialogue context and can be referenced in the utterances build inside future negotiation phases. The formal model of dialogue presented in this paper articulates in the following way: inquisitive semantics is used to model both interrogative and declarative sentences (see the grey rectangles around the utterances). Dashed lines represent dynamic phenomena: at utterance level, dynamicity allows us to compute all the semantic representations; between utterances inside a negotiation phase, dynamicity allows referencing things introduced in previous utterances. Between dialogue context and utterances, dynamicity allows to reference previously stored things. Dotted 1 ines represent the epistemic phenomena in dialogue and are, in this schema, restrained to the participants private contexts, so they are condensed on the character's heads. Full lines represent all the dialogue gameboard's updates: the ones between utterances update the utterance-related information, closing the negotiation phase triggers the computation of new dialogue context updates and the arrow between the negotiation phase and the dialogue context represents their storage. The full-line boxes encapsulating the utterances account for the updates in the question under discussion, i.e the issue that is being discussed in this negotiation phase.

\section{The dialogue gameboard}

Dialogue semantics is radically context-dependent. Following [11], we model the dialogues and dialogue context in particular using dialogue gameboards (DGB). We use one DGB per participant in order to model their private contexts, plus one DGB for the public context. A dialogue gameboard is composed of different fields:

$$
\left[\begin{array}{cc}
\text { speaker }: & \text { Individual } \\
\text { addressee }: & \text { Individual } \\
\text { FACTS : } & \text { set of propositions } \\
\text { QUD : } & \text { partially ordered set of questions }
\end{array}\right]
$$

Several are used to store information about the indexicals, typically, the speaker and the addressee. FACTS is used to store the propositions that have been agreed on by the dialogue participants in the case of public dialogue gameboards (the ones that model a shared view of the dialogue), and propositions that are personal to the participant

\footnotetext{
${ }^{1}$ https://www.nytimes.com/2015/04/15/science/a-math-problem-from-singapore-goes-viralwhen-is-cheryls-birthday.html
} 


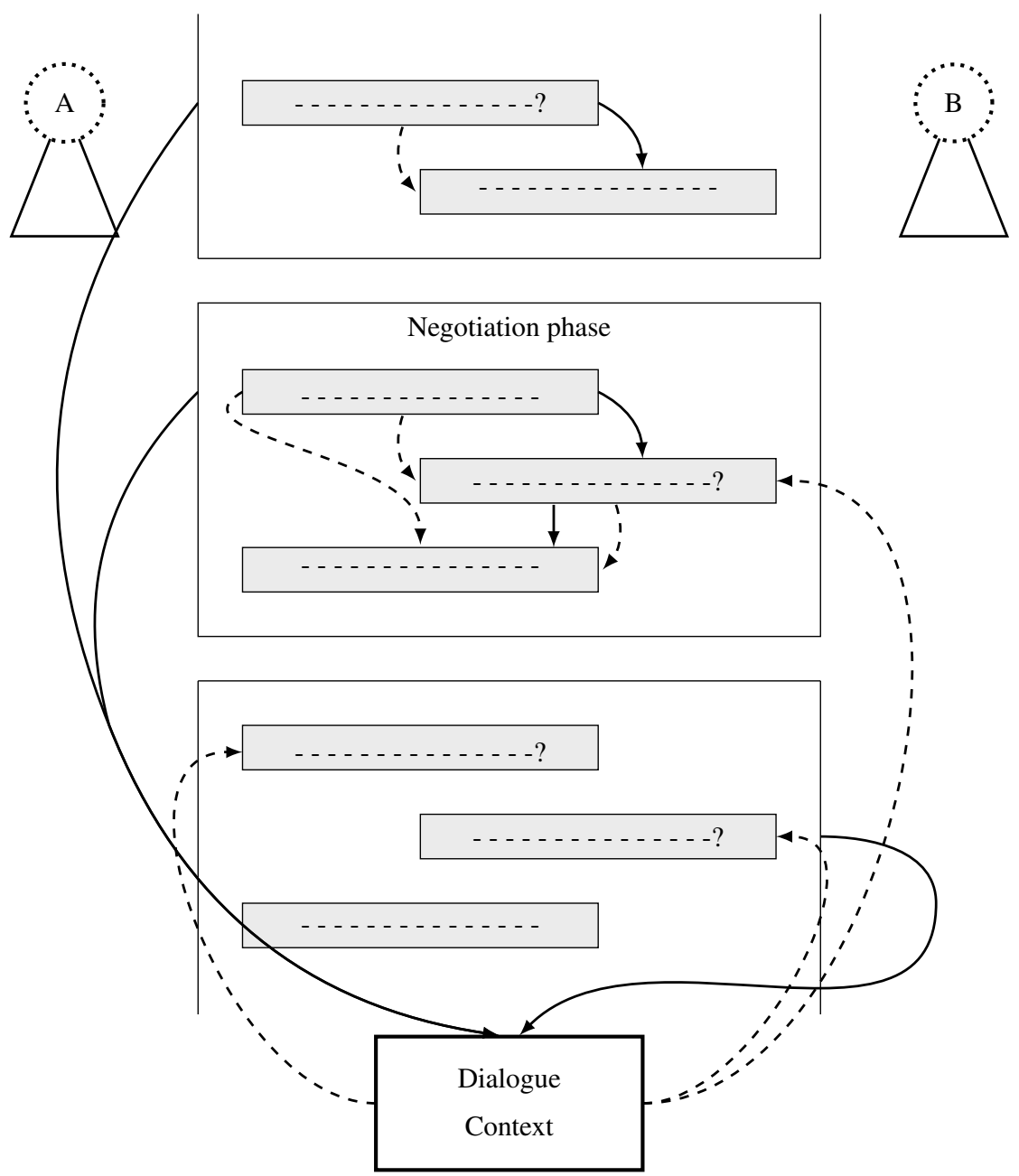

Fig. 1. Subdivision of dialogue in negotiation phases, adapted from [2].

in the case of private dialogue gameboards. QUD, which stays for questions under discussion, stores the issues that have to be solved by the dialogue participants. These issues are raised by questions asked by the dialogue participants, but also by other types of utterances, as any proposition has to be discussed before being accepted by all the participants. The $Q U D$ is a partially ordered set where the order is used to decide which issue has to be solved first if several issues are raised at the same time.

\section{Inquisitive semantics}

As pointed out in the introduction, developing a logic-based formal model of dialogue requires a logic that can express the semantic content of both declarative and interrog- 
ative sentences. This need motivates the use of inquisitive logic, which is a logic that allows for a uniform treatment of both kinds of sentences.

As opposed to traditional modal logic, where a proposition is interpreted as a set of possible worlds, inquisitive semantics interprets a proposition as a set of sets of possible worlds. Intuitively, an inquisitive proposition may therefore be seen as a set of classical (modal) propositions. This allows questions to be assigned semantics akin to Hamblin's alternative semantics [14]. Inquisitive semantics, however, differs from Hamblin's alternative semantics in several respects.

Technically, in inquisitive logic, a proposition is defined to be a non-empty set of sets of possible worlds that is downward-closed with respect to set inclusion. As a consequence, conjunction, disjunction, and entailment can be defined in a standard way, i.e., as intersection, union, and inclusion, respectively. Let us illustrate this by an example.

Suppose it is known that Cheryl's birthday is either May 15, June 17, or July 14. Accordingly, we define a set of possible worlds, $W=\left\{w_{5.15}, w_{6.17}, w_{7.14}\right\}$, where each possible world corresponds respectively to one of Cheryl's possible birthdates. Then, the proposition $\varphi_{1}$ that Cheryl is born on May 15 is interpreted as follows:

$$
\llbracket \varphi_{1} \rrbracket=\left\{\left\{w_{5.15}\right\}, \varnothing\right\}
$$

The proposition $\varphi_{2}$ that she is born on June 17 is interpreted in a similar way:

$$
\llbracket \varphi_{2} \rrbracket=\left\{\left\{w_{6.17}\right\}, \varnothing\right\}
$$

Then, the inquisitive disjunction of $\varphi_{1}$ and $\varphi_{2}$ is interpreted as the union of their interpretations:

$$
\llbracket \varphi_{1} \vee_{\mathrm{i}} \varphi_{2} \rrbracket=\left\{\left\{w_{5.15}\right\},\left\{w_{6.17}\right\}, \varnothing\right\}
$$

This disjunction does not correspond to a proposition asserting that Cheryl's birthday is either May 15 or June 17, it rather corresponds to the question whether Cheryl's birthday is May 15 or June 17, assuming that she is born at one of these two dates. The mere assertion that her birthday is either May 15 or June 17 is interpreted in a different way:

$$
\left\{\left\{w_{5.15}, w_{6.17}\right\},\left\{w_{5.15}\right\},\left\{w_{6.17}\right\}, \varnothing\right\}
$$


It is well known that intensional logic can be defined by embedding it in Gallin's Ty2 [10]. We provide here below a similar embedding for first-order inquisitive logic ${ }^{2}$ :

$$
\begin{aligned}
& \mathbf{R}_{\mathrm{i}} t_{1} \ldots t_{n}:= \mathscr{P}\left(\mathbf{R} t_{1} \ldots t_{n}\right) \\
& \text { where } \mathscr{P} a=\lambda b^{\mathbf{s} \rightarrow \mathbf{t}} \cdot \forall w^{\mathbf{s}} \cdot(b w) \rightarrow(a w) \\
& \varphi \wedge_{\mathrm{i}} \psi \quad:= \lambda a^{\mathbf{s} \rightarrow \mathbf{t}} \cdot(\varphi a) \wedge(\psi a) \\
& \varphi \vee_{\mathrm{i}} \psi:=\lambda a^{\mathbf{s} \rightarrow \mathbf{t}} \cdot(\varphi a) \vee(\psi a) \\
& \varphi \rightarrow_{\mathrm{i}} \psi \quad:= \lambda a^{\mathbf{s} \rightarrow \mathbf{t}} \cdot \forall b^{\mathbf{s} \rightarrow \mathbf{t}} \cdot\left(\forall w^{\mathbf{s}} \cdot(b w) \rightarrow(a w)\right) \rightarrow((\varphi b) \rightarrow(\psi b)) \\
& \neg_{\mathrm{i}} \varphi:=\lambda a^{\mathbf{s} \rightarrow \mathbf{t}} \cdot \forall w^{\mathbf{s}} \cdot(a w) \rightarrow \neg\left(\exists b^{\mathbf{s} \rightarrow \mathbf{t}} \cdot(\varphi b) \wedge(b w)\right) \\
& \forall_{\mathrm{i}} x^{\mathbf{e}} \cdot \varphi x \quad:=\lambda a^{\mathbf{s} \rightarrow \mathbf{t}} \cdot \forall x^{\mathbf{e}} \cdot \varphi x a \\
& \exists_{\mathrm{i}} x^{\mathbf{e}} \cdot \varphi x \quad:=\lambda a^{\mathbf{s} \rightarrow \mathbf{t}} \cdot \exists x^{\mathbf{e}} \cdot \varphi x a
\end{aligned}
$$

Inquisitive logic also features two projection operators, ! and ?. The first one transforms any proposition into a purely informative one by cancelling its inquisitive content. Conversely, the second one transforms any proposition into a purely inquisitive one by cancelling its informative content. These projection operators may be defined as follows:

$$
\begin{aligned}
& ! \varphi:=\neg_{\mathrm{i}} \neg_{\mathrm{i}} \varphi \\
& ? \varphi:=\varphi \vee_{\mathrm{i}} \neg_{\mathrm{i}} \varphi
\end{aligned}
$$

These two operators are useful to turn a question into an assertion, and vice versa. For instance, by applying ! to (4), one obtains the proposition asserting that Cheryl's birthday is either May 15 or June 17:

$$
\llbracket !\left(\varphi_{1} \vee_{\mathrm{i}} \varphi_{2}\right) \rrbracket=\left\{\left\{w_{5.15}, w_{6.17}\right\},\left\{w_{5.15}\right\},\left\{w_{6.17}\right\}, \varnothing\right\}
$$

On the other hand, by applying ? to (2), one obtains an inquisitive proposition that corresponds to the issue whether Cheryl is born on May 15 or not:

$$
\llbracket ? \varphi_{1} \rrbracket=\left\{\left\{w_{6.17}, w_{7.14}\right\},\left\{w_{5.15}\right\},\left\{w_{6.17}\right\},\left\{w_{7.14}\right\}, \varnothing\right\}
$$

Inquisitive propositions being downward-closed sets, they are completely characterized by their maximal elements. In the sequel of this paper, we will use the notation $[a, b, c, \ldots]$ to denote the downward-closure of the set $\{a, b, c, \ldots\}$. With this convention, Example (7) may rewritten as follows:

$$
\llbracket ? \varphi_{1} \rrbracket=\left[\left\{w_{6.17}, w_{7.14}\right\},\left\{w_{5.15}\right\}\right]
$$

Example (4) illustrates that an inquisitive proposition has both an informative and an inquisitive content. It is even the fact that every inquisitive proposition may be defined as the conjunction of a purely informative proposition with a purely inquisitive one. It is indeed not difficult to establish that every proposition $\varphi$ is such that:

$$
\varphi=! \varphi \wedge ? \varphi
$$

\footnotetext{
${ }^{2}$ Where $\mathbf{s}$ is the type of possible worlds, $\mathbf{t}$ is the type of truth values, $\mathbf{e}$ is the type of individuals, following [16].
} 
This fact has an interesting consequence with respect to dialogue-gameboard modeling: it allows the $Q U D$ and the FACTS to be expressed by a unique proposition, say $\varphi$, such that:

$$
\mathrm{QUD}=? \varphi \quad \text { and } \quad \mathrm{FACTS}=! \varphi
$$

We end this quick review of inquisitive semantics by showing how it can be used to provide a Montague-like compositional semantics to questions. To this end, we consider the question when Cheryl's birthday is (as it occurs in the sentence Albert does not know when Cheryl birthday is). The abstract syntax of the sentence is specified by means of the term:

$$
\text { WHEN } \left.\left(\lambda x^{N P} \text {. IS } x \text { (POSSESSIVE CHERYL BIRTHDAY }\right)\right)
$$

which is built upon the following signature:

$$
\begin{aligned}
& \text { CHERYL }: N P \\
& \text { BIRTHDAY }: N \\
& \text { POSSESSIVE }: N P \rightarrow N \rightarrow N P \\
& \text { IS }: N P \rightarrow N P \rightarrow S \\
& \text { WHEN }:(N P \rightarrow S) \rightarrow S
\end{aligned}
$$

We define $\mathbf{p}$ to be the type of inquisitive propositions, i.e., $\mathbf{p}=(\mathbf{s} \rightarrow \mathbf{t}) \rightarrow \mathbf{t}$. Then, the semantic interpretation of the syntactic categories is as follows:

$$
\begin{aligned}
\llbracket N P \rrbracket & :=(\mathbf{e} \rightarrow \mathbf{p}) \rightarrow \mathbf{p} \\
\llbracket N \rrbracket: & =\mathbf{e} \rightarrow \mathbf{p} \\
\llbracket S \rrbracket & :=\mathbf{p}
\end{aligned}
$$

In order to express the semantic interpretation of (9), we use the following non-logical constants:

$$
\begin{gathered}
\text { cheryl : } \mathrm{e} \\
\text { birthday }: \mathrm{e} \rightarrow \mathrm{s} \rightarrow \mathrm{t} \\
\quad \text { of }: \mathrm{e} \rightarrow \mathrm{e} \rightarrow \mathrm{s} \rightarrow \mathrm{t}
\end{gathered}
$$

Following the inquisitive interpretation of an atomic proposition, we raise the types of the relation symbols:

$$
\begin{aligned}
\text { birthday }_{\mathrm{i}} & :=\lambda x^{\mathrm{e}} \cdot \mathscr{P}(\text { birthday } x) \\
\text { of }_{\mathrm{i}} & :=\lambda x^{\mathrm{e}} y^{\mathrm{e}} \cdot \mathscr{P}(\text { of } x y)
\end{aligned}
$$

We also raise the equality relation between entities:

$$
\left(x={ }_{\mathrm{i}} y\right):=\mathscr{P}\left(\lambda w^{\mathbf{s}} \cdot x=y\right)
$$


Finally, we provide the lexical entries with the following interpretations:

$$
\begin{aligned}
\llbracket \text { CHERYL } \rrbracket & :=\lambda p^{\mathbf{e} \rightarrow \mathbf{p}} \cdot p \text { cheryl } \\
\llbracket \text { BIRTHDAY } \rrbracket & :=\text { birthday }_{\mathrm{i}} \\
\llbracket \text { POSSESSIVE } \rrbracket & :=\lambda p^{(\mathbf{e} \rightarrow \mathbf{p}) \rightarrow \mathbf{p}} q^{\mathbf{e} \rightarrow \mathbf{p}_{r} \mathbf{e} \rightarrow \mathbf{p}} \cdot p\left(\lambda x^{\mathbf{e}} \cdot !\left(\exists_{\mathrm{i}} y^{\mathbf{e}} \cdot(q y) \wedge_{\mathrm{i}}\left(\mathbf{o f}_{\mathrm{i}} x y\right) \wedge_{\mathrm{i}}(r y)\right)\right) \\
\llbracket \text { IS } \rrbracket & :=\lambda p^{(\mathbf{e} \rightarrow \mathbf{p}) \rightarrow \mathbf{p}(\mathbf{e} \rightarrow \mathbf{p}) \rightarrow \mathbf{p}} \cdot p\left(\lambda x^{\mathbf{e}} \cdot q\left(\lambda y^{\mathbf{e}} \cdot x=_{\mathrm{i}} y\right)\right) \\
\llbracket \text { WHEN } \rrbracket & :=\lambda p^{((\mathbf{e} \rightarrow \mathbf{p}) \rightarrow \mathbf{p}) \rightarrow \mathbf{p}} \cdot \exists_{\mathrm{i}} x^{\mathbf{e}} \cdot p\left(\lambda q^{\mathbf{e} \rightarrow \mathbf{p}} \cdot q x\right)
\end{aligned}
$$

Then, applying the above semantic recipes to term (9) yields the following results:

$$
\exists_{\mathrm{i}} x^{\mathbf{e}} \cdot !\left(\exists_{\mathrm{i}} y^{\mathbf{e}} \cdot\left(\text { birthday }_{\mathrm{i}} y\right) \wedge_{\mathrm{i}}\left(\text { of }_{\mathrm{i}} \text { cheryl } y\right) \wedge_{\mathrm{i}}\left(x==_{\mathrm{i}} y\right)\right)
$$

\section{Dialogue participant's epistemic state}

As explained in the introduction, the short exchange between Cheryl and Albert in (1) exemplifies the epistemic nature of a (cooperative) dialogue, and demonstrates the need to represent the knowledge states of the dialogue participants. Consequently, we must add to the dialogue context some information that models the private knowledge of each agent (i.e., each dialogue participant). ${ }^{3}$

Following Ciardelli's and Roelofsen's [5], we associate to each agent a and each possible world $w$ an inquisitive proposition $\Sigma_{\mathbf{a}, w}$ that models the epistemic and inquisitive state of agent a at world $w$. In type-theoretic terms, this may be modeled by a function $\Sigma$ of type $\mathbf{e} \rightarrow \mathbf{s} \rightarrow \mathbf{p}$. The epistemic modality associated to agent $\mathbf{a}$ is then defined as follows:

$$
\mathrm{K} \mathbf{a} \varphi:=\lambda q^{\mathbf{s} \rightarrow \mathbf{t}} \cdot \forall w^{\mathbf{s}} .(q w) \rightarrow(\varphi(\bigcup(\Sigma \mathbf{a} w)))
$$

where $\bigcup S=\lambda x . \exists a .(S a) \wedge(a x)$.

Let us now continue the example started in the previous section by showing how to interpret the sentence Albert does not know when Cheryl birthday is. Its abstract syntax is given by the term:

$$
\text { NOT (KNOW (WHEN } \left.\left.\left(\lambda x^{N P} \text {. IS } x \text { (POSSESSIVE CHERYL BIRTHDAY }\right)\right)\right) \text { ) ALBERT (11) }
$$

where in addition to the already defined abstract syntactic constants, we have:

$$
\begin{aligned}
\text { ALBERT } & : N P \\
\text { KNOW }: & S \rightarrow N P \rightarrow S \\
\text { NOT }: & (N P \rightarrow S) \rightarrow N P \rightarrow S
\end{aligned}
$$

\footnotetext{
${ }^{3}$ In [11], the dialogue context includes, in addition to the common dialogue gameboard, private dialogue gameboards, one for each agent. Our approach is slightly different. What we model is not quite the private knowledge of each agent but rather what is commonly known about this private knowledge.
} 
The semantic interpretations of these new lexical entries is then as follows:

$$
\begin{aligned}
\llbracket \text { ALBERT } \rrbracket & :=\lambda p^{\mathbf{e} \rightarrow \mathbf{p}} \cdot p \text { albert } \\
\llbracket \text { KNOW } \rrbracket & :=\lambda p^{\mathbf{p}} q^{(\mathbf{e} \rightarrow \mathbf{p}) \rightarrow \mathbf{p}} \cdot q\left(\lambda x^{\mathbf{e}} \cdot \mathbf{K} x p\right) \\
\llbracket \text { NOT } \rrbracket & :=\lambda p^{((\mathbf{e} \rightarrow \mathbf{p}) \rightarrow \mathbf{p}) \rightarrow \mathbf{p}} q^{(\mathbf{e} \rightarrow \mathbf{p}) \rightarrow \mathbf{p}} \cdot q\left(\lambda x^{\mathbf{e}} . \neg_{\mathrm{i}}\left(p\left(\lambda r^{\mathbf{e} \rightarrow \mathbf{p}} \cdot r x\right)\right)\right)
\end{aligned}
$$

With these entrie interpretations, we obtain the expected interpretation of (11):

$$
\neg_{\mathrm{i}}\left(\operatorname{Kalbert}\left(\exists_{\mathrm{i}} x^{\mathbf{e}} . !\left(\exists_{\mathrm{i}} y^{\mathbf{e}} .\left(\text { birthday }_{\mathrm{i}} y\right) \wedge_{\mathrm{i}}\left(\text { of }_{\mathbf{i}} \operatorname{cheryl} y\right) \wedge_{\mathrm{i}}\left(x==_{\mathrm{i}} y\right)\right)\right)\right)
$$

\section{Dialogue dynamics and context updating}

As we have seen, inquisitive logic allows one to assign a formal semantics to each dialogue turn in a compositional way akin to Montague's [6]. The next step is to provide our model with some dynamics that will allow a dialogue turn to update the current dialogue context. For this purpose, we adapt the type-theoretic dynamic logic introduced in [7] and further developed in [15]. This approach has several advantages. It allows several dynamic phenomena to be integrated in a same framework (typically, discourse dynamic, as in [13,9], and epistemic dynamic as in [8,5]). It also allows for a treatment of dynamics at a subsentential level (as in [17]). ${ }^{4}$

The first question to settle is how to model dialogue contexts. We have seen that a typical dialogue gameboard consists of the speaker, the addressee, the FACTS, and the $Q U D$. We have also seen that both the FACTS and the QUD may be encoded as a single inquisitive proposition. In addition, a dialogue context must also contain information about the private knowledge of the dialogue participants. Accordingly, we define a dialogue context to be a 4-tuple $(s, a, Q, K)$ where:

- $s$, which is of type e, is the speaker;

- $a$, which is of type $\mathbf{e}$, is the addressee;

- $Q$, which is of type $\mathbf{p}$, is an inquisitive proposition that models both the FACTS and the $Q U D$;

- $K$, which is of type $\mathbf{e} \rightarrow \mathbf{s} \rightarrow \mathbf{p}$, is the function that associates to each agent their epistemic state at a given possible world.

Let $\mathbf{d}=\mathbf{e} \times \mathbf{e} \times \mathbf{p} \times(\mathbf{e} \rightarrow \mathbf{s} \rightarrow \mathbf{p})$ be the type of dialogue contexts. We posit the existence of four context accessing functions:

$$
\begin{aligned}
\text { speaker }: \mathrm{d} & \rightarrow \mathrm{e} \\
\text { addresse }: \mathrm{d} & \rightarrow \mathrm{e} \\
\text { qud }: \mathrm{d} & \rightarrow \mathrm{p} \\
\Sigma: \mathrm{d} & \rightarrow \mathrm{e} \rightarrow \mathrm{s} \rightarrow \mathrm{p}
\end{aligned}
$$

\footnotetext{
${ }^{4}$ For the sake of conciseness and simplicity, in this paper, we give a simplified version that does not allow for anaphora resolution. This simplification dispenses one from modeling the socalled right context using a continuation. Taking anaphora resolution into account is feasible but involves a lot of technical details that are orthogonal to our main concern.
} 
which simply correspond to projection operators.

Given the notion of a dynamic context, a dynamic proposition is defined to be an inquisitive proposition depending upon such a context. Hence, we define $\mathbf{P}=\mathbf{d} \rightarrow \mathbf{p}$ to be the type of dynamic propositions. The interpretation of the logical connectives and quantifiers must then be changed in order to accommodate dynamic propositions. The new interpretation is as follows:

$$
\begin{array}{ll}
\mathbf{R}_{\mathrm{d}} t_{1} \ldots t_{n}:=\lambda c^{\mathrm{d}} \cdot\left(\mathbf{R}_{\mathrm{i}} t_{1} \ldots t_{n}\right) \wedge_{\mathrm{i}} !(\mathbf{q u d} c) \\
\varphi \wedge_{\mathrm{d}} \psi & :=\lambda c^{\mathbf{d}} \cdot(\varphi c) \wedge_{\mathrm{i}}(\psi c) \\
\varphi \vee_{\mathrm{d}} \psi & :=\lambda c^{\mathrm{d}} \cdot(\varphi c) \vee_{\mathrm{i}}(\psi c) \\
\varphi \rightarrow_{\mathrm{d}} \psi & :=\lambda c^{\mathrm{d}} \cdot(\varphi c) \rightarrow_{\mathrm{i}}(\psi c) \\
\neg_{\mathrm{d}} \varphi & :=\lambda c^{\mathrm{d}} \cdot \neg_{\mathrm{i}}(\varphi c) \\
\forall_{\mathrm{d}} x^{\mathbf{e}} \cdot \varphi x & :=\lambda c^{\mathrm{d}} \cdot \forall_{\mathrm{i}} x^{\mathbf{e}} \cdot \varphi x c \\
\exists_{\mathrm{d}} x^{\mathbf{e}} \cdot \varphi x & :=\lambda c^{\mathrm{d}} \cdot \exists_{\mathrm{i}} x^{\mathbf{e}} \cdot \varphi x c \\
!_{\mathrm{d}} \varphi & :=\lambda c^{\mathrm{d}} \cdot !(\varphi c) \\
?_{\mathrm{d}} \varphi & :=\lambda c^{\mathrm{d}} \cdot ?(\varphi c) \\
\mathrm{K}_{\mathrm{d}} \mathbf{a} \varphi & :=\lambda c^{\mathrm{d}} \cdot \lambda q^{\mathbf{s} \rightarrow \mathbf{t}} \cdot\left(\forall w^{\mathbf{s}} \cdot(q w) \rightarrow(\varphi c(\bigcup(\Sigma c \mathbf{a} w))) \wedge(!(\mathbf{q u d} c) q)\right.
\end{array}
$$

Note how the interpretation of an atomic proposition is now sensitive to the context because it is intersected with the current FACTS. Thus, if the context establishes that Cheryl's birthday is either May 15 or June 17, the question of when is Cheryl's birthday will be equivalent to the question of whether Cheryl's birthday is May 15 or June 17. Remark that the interpretation of the epistemic modality is also sensitive to the context.

Using the dynamic logic, it is now possible to provide an interpretation to the sentence I don't know when your birthday is. To this end, we interpret the syntactic categories dynamically:

$$
\begin{aligned}
\llbracket N P \rrbracket & :=(\mathbf{e} \rightarrow \mathbf{P}) \rightarrow \mathbf{P} \\
\llbracket N \rrbracket & :=\mathbf{e} \rightarrow \mathbf{P} \\
\llbracket S \rrbracket & :=\mathbf{P}
\end{aligned}
$$

The lexical entries are kept unchanged except that the atomic propositions and the logical connectives are interpreted dynamically. For instance, we now have:

$$
\begin{aligned}
\llbracket \text { BIRTHDAY } \rrbracket & :=\text { birthday }_{\mathrm{d}} \\
\llbracket \text { WHEN } \rrbracket & :=\lambda p^{(\mathbf{e} \rightarrow \mathbf{P}) \rightarrow \mathbf{P}) \rightarrow \mathbf{P}} \cdot \exists_{\mathrm{d}} x^{\mathbf{e}} \cdot p\left(\lambda q^{\mathbf{e} \rightarrow \mathbf{P}} \cdot q x\right)
\end{aligned}
$$

Then, we may extend our semantic lexicon as follows:

$$
\begin{gathered}
\llbracket \mathrm{I} \rrbracket:=\lambda p^{\mathbf{e} \rightarrow \mathbf{P}} \cdot \lambda c^{\mathbf{d}} \cdot p(\text { speaker } c) c \\
\llbracket \mathrm{YOU} \rrbracket:=\lambda p^{\mathbf{e} \rightarrow \mathbf{P}} \cdot \lambda c^{\mathbf{d}} \cdot p(\text { addressee } c) c
\end{gathered}
$$


It remains to show how a dialog turn acts on the dialogue context. To this end, we define the following updating functions:

$$
\begin{aligned}
& \text { sets }:=\lambda c^{\mathbf{d}} x^{\mathbf{e}} \cdot(x, \text { addressee } c, \text { qud } c, \Sigma c) \quad \text { sets the speaker } \\
& \text { seta }:=\lambda c^{\mathbf{d}} x^{\mathbf{e}} .(\text { speaker } c, x, \text { qud } c, \Sigma c) \quad \text { sets the addressee } \\
& \text { upd }:=\lambda c^{\mathbf{d}} a^{\mathbf{P}} .\left(\text { speaker } c, \text { addressee } c,(\text { qud } c) \wedge_{\mathrm{i}}(a c), \lambda x^{\mathbf{e}} w^{\mathbf{s}} \cdot(\Sigma c x w) \wedge(a c)\right)
\end{aligned}
$$

We then define a dialogue turn to be a triple $(s, a, \varphi)$, of type $\mathbf{e} \times \mathbf{e} \times \mathbf{P}$, where $s$ is the speaker, $a$ is the addressee, and $\varphi$ is a dynamic proposition that expresses the semantics of the dialogue turn. Finally, we define the action on a dialogue context $C$ of such a dialogue turn as follows:

$$
C \circ(s, a, \varphi)=\operatorname{upd}(\operatorname{seta}(\operatorname{sets} c s) a) \varphi
$$

\section{A complete example: Cheryl's birthday}

Let us illustrate the way our model works by applying it to the logical puzzle known as "When is Cheryl's Birthday". Here is the wording of the problem as it appeared on the New York Times website in April 2015. ${ }^{5}$

(2) [CONTEXT: Albert and Bernard just met Cheryl. "When's your birthday?" Albert asked Cheryl. Cheryl thought a second and said, "I'm not going to tell you, but I'll give you some clues." She wrote down a list of 10 dates:

$$
\begin{aligned}
& \text { May 15, May 16, May 19, } \\
& \text { June 17, June 18 } \\
& \text { July 14, July } 16 \\
& \text { August 14, August 15, August } 17
\end{aligned}
$$

"My birthday is one of these," she said. Then Cheryl whispered in Albert's ear the month — and only the month — of her birthday. To Bernard, she whispered the day, and only the day.]

a. Cheryl (to Albert): Can you figure it out now?

b. Albert: I don't know when your birthday is, but I know Bernard doesn't know, either.

c. Bernard: I didn't know originally, but now I do.

d. Albert: Well, now I know, too!

When is Cheryl's birthday?

In order to solve the problem, the first task is to formalize the initial dialogue context. To this end, we could first define a first-order object language. This language would include atomic propositions such as May15, Jun17, Jul14, etc. (with the obvious intended meanings). Then, we would have to posit meaning postulates such as $\neg_{\mathrm{i}}\left(\right.$ May15 $\wedge_{\mathrm{i}}$ Jun17), $\neg_{\mathrm{i}}\left(\right.$ May15 $\wedge_{\mathrm{i}}$ Jun17), etc. By following such an approach,

\footnotetext{
5 https://www.nytimes.com/2015/04/15/science/a-math-problem-from-singapore-goes-viralwhen-is-cheryls-birthday.html
} 
we would model the $Q U D$ as a formula expressing the inquisitive disjunction of the possible birthdate:

$$
\text { May15 } \vee_{\mathrm{i}} \mathbf{J u n} 17 \vee_{\mathrm{i}} \mathbf{J u l 1 4} \vee_{\mathrm{i}} \text { etc }
$$

As our objective is to make our explanation clear by not overcharging them with too much syntactic details, we prefer to leave the object language implicit and reason in semantic terms with possible worlds. The set of possible world is defined in such a way that each world corresponds to a possible birthdate:

$$
W=\left\{w_{5.15}, w_{5.16}, w_{5.19}, w_{6.17}, w_{6.18}, w_{7.14}, w_{7.16}, w_{8.14}, w_{8.15}, w_{8.17}\right\}
$$

Then the initial $Q U D$, in its semantic version, corresponds to the following inquisitive proposition:

$$
\begin{aligned}
Q_{0}=[ & \left\{w_{5.15}\right\},\left\{w_{5.16}\right\},\left\{w_{5.19}\right\},\left\{w_{6.17}\right\},\left\{w_{6.18}\right\} \\
& \left.\left\{w_{7.14}\right\},\left\{w_{7.16}\right\},\left\{w_{8.14}\right\},\left\{w_{8.15}\right\},\left\{w_{8.17}\right\}\right]
\end{aligned}
$$

Let us now concentrate on the modeling of the knowledge of the agents. Consider, for instance, world $w_{5.15}$. In this world, the month of Cheryl's birthdate is May, and Albert knows it. Albert is therefore in an inquisitive state where he wonders what is the day of Cheryl's birthdate, knowing that it is either the 15th, the 16th, or the 19th. This inquisitive state is represented by the following proposition:

$$
\left[\left\{w_{5.15}\right\},\left\{w_{5.16}\right\},\left\{w_{5.19}\right\}\right]
$$

Continuing this line of reasoning, we obtain that Albert's knowledge is modeled by the following map:

$$
K_{0} \text { albert }= \begin{cases}w_{5.15}\left|w_{5.16}\right| w_{5.19} & \mapsto\left[\left\{w_{5.15}\right\},\left\{w_{5.16}\right\},\left\{w_{5.19}\right\}\right] \\ w_{6.17} \mid w_{6.18} & \mapsto\left[\left\{w_{6.17}\right\},\left\{w_{6.18}\right\}\right] \\ w_{7.14} \mid w_{7.16} & \mapsto\left[\left\{w_{7.14}\right\},\left\{w_{7.16}\right\}\right] \\ w_{8.14}\left|w_{8.15}\right| w_{8.17} & \mapsto\left[\left\{w_{8.14}\right\},\left\{w_{8.15}\right\},\left\{w_{8.17}\right\}\right]\end{cases}
$$

Similarly for Bernard:

$$
K_{0} \text { bernard }= \begin{cases}w_{7.14} \mid w_{8.14} & \mapsto\left[\left\{w_{7.14}\right\},\left\{w_{8.14}\right\}\right] \\ w_{5.15} \mid w_{8.15} & \mapsto\left[\left\{w_{5.15}\right\},\left\{w_{8.15}\right\}\right] \\ w_{5.16} \mid w_{7.16} & \mapsto\left[\left\{w_{5.16}\right\},\left\{w_{7.16}\right\}\right] \\ w_{6.17} \mid w_{8.17} & \mapsto\left[\left\{w_{6.17}\right\},\left\{w_{8.17}\right\}\right] \\ w_{6.18} & \mapsto\left[\left\{w_{6.18}\right\}\right] \\ w_{5.19} & \mapsto\left[\left\{w_{5.19}\right\}\right]\end{cases}
$$

As for Cheryl, her knowledge (which is irrelevant for the example) corresponds to the map that assigns to each world $w$ the proposition $[\{w\}]$. Our initial context is therefore $C_{0}=\left(Q_{0}, K_{0}\right) .{ }^{6}$. Let us now consider the dialogue turns. The first one, (2-a), simply

\footnotetext{
${ }^{6}$ we leave implicit the speaker and the addressee
} 
restates the $Q U D$ and does not affect the context. We therefore have $C_{1}=C_{0}$. The second turn, (2-b), is more interesting. It is interpreted as the dynamic proposition

$$
\neg_{\mathrm{d}}\left(\mathrm{K}_{\mathrm{d}} \text { albert } \varphi\right) \wedge\left(\mathrm{K}_{\mathrm{d}} \text { albert } \neg_{\mathrm{d}}\left(\mathrm{K}_{\mathrm{d}} \text { bernard } \varphi\right)\right)
$$

$\phi$ corresponds to a dynamic proposition that amounts to the QUD when evaluated with respect to the current context. It is not difficult to see that the first conjunct, when evaluated with respect to the current context, yields $\mathscr{P}(W)$, i.e., the always true proposition. The second conjunct is more interesting. When evaluated with respect to $C_{1},\left(\mathrm{~K}_{\mathrm{d}}\right.$ bernard $\left.\varphi\right)$ is interpreted as $\left[\left\{w_{6.18}, w_{5.19}\right\}\right]$. Hence, $\neg_{\mathrm{d}}\left(\mathrm{K}_{\mathrm{d}}\right.$ bernard $\left.\varphi\right)$ is interpreted as $\left[\left\{w_{5.15}, w_{5.16}, w_{6.17}, w_{7.14}, w_{7.16}, w_{8.14}, w_{8.15}, w_{8.17}\right\}\right]$. And finally, $\mathrm{K}_{\mathrm{d}}$ albert $\neg_{\mathrm{d}}\left(\mathrm{K}_{\mathrm{d}}\right.$ bernard $\left.\varphi\right)$ is interpreted as $\left[\left\{w_{7.14}, w_{7.16}, w_{8.14}, w_{8.15}, w_{8.17}\right\}\right]$. Then, updating the context $C_{1}$ yields a context $C_{2}=\left(Q_{2}, K_{2}\right)$ where:

$$
\begin{aligned}
& Q_{2}=\left[\left\{w_{7.14}\right\},\left\{w_{7.16}\right\},\left\{w_{8.14}\right\},\left\{w_{8.15}\right\},\left\{w_{8.17}\right\}\right] \\
& K_{2} \text { albert }= \begin{cases}w_{7.14} \mid w_{7.16} & \mapsto\left[\left\{\left\{w_{7.14}\right\},\left\{w_{7.16}\right\}\right]\right. \\
w_{8.14}\left|w_{8.15}\right| w_{8.17} & \mapsto\left[\left\{w_{8.14}\right\},\left\{w_{8.15}\right\},\left\{w_{8.17}\right\}\right] \\
- & \mapsto[\varnothing]\end{cases} \\
& K_{2} \text { bernard }=\left\{\begin{aligned}
w_{7.14} \mid w_{8.14} & \mapsto\left[\left\{w_{7.14}\right\},\left\{w_{8.14}\right\}\right] \\
w_{5.15} \mid w_{8.15} & \mapsto\left[\left\{w_{8.15}\right\}\right] \\
w_{5.16} \mid w_{7.16} & \mapsto\left[\left\{w_{7.16}\right\}\right] \\
w_{6.17} \mid w_{8.17} & \mapsto\left[\left\{w_{8.17}\right\}\right] \\
- & \mapsto[\varnothing]
\end{aligned}\right.
\end{aligned}
$$

By applying the same analysis to the other dialogue turns, one obtains successively the following QUD: $Q_{3}=\left[\left\{w_{7.16}\right\},\left\{w_{8.15}\right\},\left\{w_{8.17}\right\}\right]$ and $\left.Q_{4}=\left[\left\{w_{8.15}\right\}\right\}\right]$, which settles the problem.

\section{Comparision with previous work}

We conclude our paper by discussing our model and comparing it to related approaches. [8] presents Dynamic Epistemic Logic (DEL). In DEL, situations are described through sets of agents, each with individual available states of information. Then, as agents perform actions, DEL gives a way to describe the changes in the state of available information, for each agent.

Growing on DEL with an inquisitive take, [5] introduces Inquisitive Dynamic Epistemic Logic (IDEL), a framework designed to provide tools that can be used to model the information exchange between a set of agents as a dynamic process through raising and solving of issues. The approach taken in the paper chooses a bi-categorial presentation of Inquisitive Semantics, with a strict separation between interrogatives and declarative sentences. The authors reference [3] for a meaning-preserving translations between this presentation and the one we use, where interrogatives and declarative sentences are modeled as the same type of objects. In IDEL, issues are raised when the 
agents ask questions and resolved when they make assertions. This is quite orthogonal to the vision of dialogue defended by Ginzburg in KoS framework [12] and that we follow here, where every speech act gives rise to a QUD, which corresponds to an issue.

IDEL is designed "under the assumption that an agent's information is always truthfuf". Though the example we show here does not illustrate this, our model is designed with the clear objective of working with real-life data and therefore in settings where disagreements can and do occur. Participants may reject an asserted fact. The negotiation phases model adds a protective additional step in the computation of the dialogue representations that bypasses this issue in a direct way. [5] suggests to try using weaker epistemic modalities such as belief and allowing disagreement to occur in order to address this difficulty.

The last section of [5] draws a comparison between IDEL and Dynamic Epistemic Logic with Questions (DELQ), as presented in [1]. DELQ is based on epistemic models enriched with a set of issues, one per agent. Then, dynamicity is added through a several actions, of which we focus on two: public announcement "that $\phi$ is the case" and public asking "whether $\phi$ is the case".

Thus, in DELQ all the sentences are considered to be declarative, none are treated as syntactically interrogative or semantically inquisitive [5]. The difference between questions and assertions is drawn through dynamic actions, at the speech act level. In IDEL, the difference between questions and assertions exists at the syntactic level through the form of interrogative sentences. In our approach, the difference between questions and assertions is acknowledged at the syntactic level but is smoothed in the semantics, as we represent issues and propositions as the same type of objects.

[5] concludes on the need to investigate a dynamic epistemic version of [4], the version of Inquisitive Semantics we presented in Section 3. This article presents our take at this investigation. We do not claim here that our model works better than IDEL, our idea actually comes from a different perspective: starting from dialogue studies and taking an orientation towards real-life data modeling.

\section{Conclusion}

Our approach grows from linguistic considerations of interrogative and declarative sentences as speech acts. We take roots in [11] but also in the syntactic parses of the speech acts in order to build our representations. The model presented in this paper addresses phenomena related to context-managing but also to dialogue management, through the way utterances influence public knowledge of private contexts. We harmonically combine several frameworks in order to model complex dialogical interactions in a logically sound way. Solving Cheryl's birthday puzzle gives us a proof of concept for the possibilities of logical reasoning through dialogue modelling. Inquisitive Semantics provides a uniform way of modeling interrogative and declarative sentences, which we think to be of the greatest importance when dealing with dialogue modelling, especially in a real-life data perspective. Next, our model needs to be scaled up in order to be applied to bigger and more complex dialogues. We hope to achieve that through the articulation of negotiation phases. In this paper, we bypassed several linguistic and logical problems related to tense and modality; future work should take these into account. Another in- 
teresting research direction would be to compare the way our model behaves on English with other, especially non-Indo-European, languages.

\section{References}

1. J. Van Benthem and Ş. Minică. Toward a dynamic logic of questions. Journal of Philosophical Logic, 41(4):633-669, 2012.

2. M. Boritchev and M. Amblard. Picturing Questions and Answers - a formal approach to SLAM. In (In)coherence of discourse - Formal and Conceptual issues of Language. Springer, 2019. Language, Cognition and Mind.

3. I. Ciardelli, J. Groenendijk, and F. Roelofsen. On the semantics and logic of declaratives and interrogatives. Synthese, 192(6):1689-1728, 2015.

4. I. Ciardelli, J. Groenendijk, and F. Roelofsen. Inquisitive Semantics. Oxford Surveys in Semantics and Pragmatics. Oxford University Press, 2018.

5. I. Ciardelli and F. Roelofsen. Inquisitive dynamic epistemic logic. Synthese, 40(6):16431687, 2015.

6. I. Ciardelli, F. Roelofsen, and N. Theiler. Composing alternatives. Linguistic \& Philosophy, 40:1-36, 2017.

7. Ph. de Groote. Towards a Montagovian Account of Dynamics. In Proceedings of Semantics and Linguistic Theory XVI, pages 1-16. Cornell University, Ithaca, NY, 2006.

8. H. Van Ditmarsch, W. van Der Hoek, and B. Kooi. Dynamic epistemic logic, volume 337 of Synthese Library. Springer, 2008.

9. J. Dotlačil and F. Roelofsen. Dynamic inquisitive semantics: Anaphora and questions. In M.T. Espinal, E. Castroviejo, M. Leonetti, L. McNally, and C. Real-Puigdollers, editors, Proceedings of Sinn und Bedeutung 23, volume 1, pages 365-382. Universitat Autònoma de Barcelona, Bellaterra (Cerdanyola del Vallès), 2019.

10. Daniel Gallin. Intensional and Higher-Order Modal Logic, volume 19 of Mathematics Studies. North-Holland, 1975.

11. J. Ginzburg. The interactive stance. Oxford University Press, 2012.

12. J. Ginzburg. Semantics of dialogue. The Cambridge Handbook of Formal Semantics, 2016.

13. J. Groenendijk and M. Stokhof. Dynamic predicate logic. Linguistic \& Philosophy, 14(1):39-100, 1991.

14. C .L. Hamblin. Questions in Montague English. Foundations of Language, 10(1):41-53, 1973.

15. E. Lebedeva. Expressing discourse dynamics through continuations. Thèse de doctorat, Université de Lorraine, 2012.

16. R. Montague. The proper treatment of quantification in ordinary english. In Approaches to natural language, pages 221-242. Springer, 1973.

17. R. Muskens. Combining Montague semantics and discourse representation. Linguistics and Philosophy, 19:143-186, 1995.

18. R. Stalnaker. Common ground. Linguistics and philosophy, 25(5-6):701-721, 2002. 